REDES- Revista hispana para el análisis de redes sociales

Vol.15, \#1, Diciembre 2008

http: // revista-redes. rediris.es

\title{
El hogar en red ${ }^{1}$
}

\author{
Tracy L. M. Kennedy \& Barry Wellman², NetLab, University of Toronto
}

\section{Resumen}

Proponemos que los individuos, en lugar de las solidaridades familiares, se han convertido en la principal unidad de conectividad en el hogar. Muchos hogares no funcionan como grupos tradicionales densamente conectados sino como redes sociales más dispersas donde los individuos tratan de hacer compatibles sus dispares horarios y agendas. En un momento en el que muchas personas ejercen múltiples roles individuales en casa, en la comunidad y en el trabajo nos preguntamos: ¿cómo se comunican entre sí los adultos miembros del hogar? ¿Cómo utilizan los adultos miembros del hogar las tecnologías de la información y la comunicación (TICs) para organizar, comunicar y coordinar su tiempo libre y su comportamiento social dentro y fuera de casa? Las entrevistas y encuestas realizadas en 2004-2005 en East York (Toronto, Canadá) muestran que los hogares siguen conectados - pero como redes más que como grupos solidarios. Describimos cómo los individuos en red construyen puentes entre sus relaciones y se conectan entre sí dentro y fuera de casa. Las TICs han permitido a los miembros del hogar la capacidad de seguir sus caminos separados mientras permanecen conectados - por teléfono móvil, correo electrónico e IM, así como con las líneas telefónicas tradicionales. De ese modo, en lugar de desmembrar las familias, las TICs con frecuencia facilitan la comunicación, las relaciones y la integración funcional.

Palabras clave: Hogar - TICS - Redes Sociales - Integración Familiar.

\begin{abstract}
We argue that individuals, rather than family solidarities, have become the primary unit of household connectivity. Many households do not operate as traditional denselyknit groups but as more sparsely-knit social networks where individuals juggle their somewhat separate agendas and schedules. At a time when many people enact multiple, individual roles at home, in the community and at work, we ask: how do adult household members communicate with each other? How do adult household members use information and communication technologies (ICTs) to organize, communicate and coordinate their leisure and social behaviour both inside and outside the home? Interviews and surveys conducted in 2004-2005 in the Toronto, Canada area of East York show that households remain connected - but as networks rather than solidary groups. We describe how networked individuals bridge their relationships

\footnotetext{
${ }^{1}$ Publicado originalmente en: Information, Communication and Society (2007), 10 (5), 645-670. Traducción de Isidro Maya J ariego.

${ }^{2}$ Nuestro agradecimiento a los demás miembros del equipo de Vidas Conectadas: Kristen Berg, Bernie Hogan, J effrey Boase, Juan Antonio Carrasco, Rochelle Côté y Jennifer Kayahara, que junto con los autores desarrollaron la encuesta y los guiones de entrevista y realizaron las entrevistas. También nos hemos beneficiado del consejo de Wenhong Chen, Paul DiMaggio, Bonnie Erickson, Inna Romanovska, Irina Shklovski, Beverly Wellman, Sandy Welsh, y del East York's Neighbourhood Information Centre. Agradecemos la ayuda de nuestros directivos, encuestadores, procesadores de datos y transcriptores (listados en http://www.chass.utoronto.ca/ wellman). Agradecemos el apoyo financiero de Intel Research,
} Microsoft Research, el Social Sciences and Humanities Research Council of Canada, y Bell University Labs.
\end{abstract}


and connect with each other inside and outside the home. ICTs have afforded household members the ability to go about on their separate ways while staying more connected - by mobile phone, email and IM- as well as by traditional landlines. In such ways, rather than pulling families apart, ICTs often facilitate communication, kinship and functional integration.

Key words: Household - ICT - Social Networks - Family Integration.

\section{Lejos de Pleasantville: Hogares doblemente en red}

Cuando Hillary Clinton afirmó "hace falta un pueblo para criar a un niño" (1996), estaba incidiendo de un modo admirable en la implicación de las redes sociales en las relaciones del hogar. Nosotros vamos más allá para afirmar que los hogares contemporáneos están doblemente en red.

1. Están densamente conectados por los nuevos y los viejos medios de comunicación: teléfonos (líneas fijas y móviles) e Internet (correo electrónico y mensajería instantánea).

2. Los propios hogares son con frecuencia redes individualizadas en lugar de grupos solidarios. Cada miembro del hogar funciona como un actor semiautónomo, con su propia agenda, y utilizando gran variedad de medios de comunicación y de transporte para contactar y coordinarse entre sí.

Aunque esta tendencia hacia hogares en red empezara antes de la proliferación de Internet y del teléfono móvil, la naturaleza intrínsecamente individual de estos medios - en contraste con las llamadas a los teléfonos del hogar y las visitas en persona a casa- ha facilitado la transmutación de las hogares en redes, igual que los coches y los teléfonos han llevado a que las comunidades personales sean redes sociales dispersas en lugar de barrios y ciudades delimitados localmente (Wellman, 2001).

Con una capacidad de previsión pionera, Elizabeth Bott afirmaba hace más de cincuenta años que "ninguna familia urbana podría sobrevivir sin su red de relaciones externas" (1957; p. 281). Ella mostró que los hogares en red tenían diferentes tipos de relaciones con los familiares que los hogares solidarios. Desde entonces, la organización del hogar ha ido cambiando hacia agendas y trayectorias más individuales -incluso antes de la revolución de las TICS. Mientras que los matrimonios o las parejas de hecho con hijos suponían el 55 por ciento de los hogares canadienses en 1981, sólo suponen el 44 por ciento en 2001 (Statistics Canada, 2001). Del mismo modo, la prevalencia de las parejas de hecho (parejas no casadas que cohabitan a largo plazo) 
ha aumentado: frente a un 6 por ciento de las parejas en 2001, se han más que duplicado hasta un 14 por ciento en 2001 (Statistics Canada, 2001).

Los hogares se han vuelto menos estables en composición y en relaciones de rol. Mientras que la tasa de divorcio en Canadá se ha mantenido constante en torno al 38 por ciento a lo largo de los últimos años, el porcentaje de reincidencia en el divorcio para el caso de mujeres divorciadas que vuelven a casarse se ha triplicado, de un 5 por ciento en 1973 hasta el 15 por ciento en 2003 (Statistics Canada, 2003a). De un modo similar, la tasa de divorcio de hombres previamente casados se ha más que triplicado, desde el 5 por ciento en 1973 hasta el 16 por ciento en 2003.

Los hogares se han vuelto más pequeños, haciendo que la necesidad de un control centralizado sea menos crucial. Los hogares de personas solas han aumentado progresivamente desde el 20 por ciento en 1981 hasta el 33 por ciento en 2001. En 2001 había prácticamente tantos hogares de una persona como hogares de cuatro o más personas (Statistics Canada, 2001). Los hogares de dos personas también han aumentado desde el 29 por ciento en 1981 hasta el 33 por ciento en 2001. El tipo de hogar que crece más rápidamente en Canadá en 2001 consiste normalmente en una pareja sin hijos o en una madre sola con un hijo. Las parejas sin hijos han aumentado desde el 34 por ciento de los hogares en 1981 y el 38 por ciento en 1991 hasta el 41 por ciento en 2001 (Statistics Canada, 2001; 2005).

Por tanto, no es sorprendente que los hogares canadienses contengan menos niños que antes. El descenso en el tamaño del hogar se relaciona parcialmente con el hecho de que las mujeres tengan menos hijos (o no tengan), con un descenso en la tasa de natalidad desde 1.6 niños por mujer en el período 1976-1981 hasta 1.5 niños por mujer en el período 1996-2001. Un mayor porcentaje de jóvenes adultos están posponiendo el momento de tener hijos para seguir sus intereses educativos y establecer sus carreras.

Con un 73 por ciento de los hombres y un 62 por ciento de las mujeres participando en la fuerza de trabajo (Statistics Canada, 2005), más hogares corresponden a parejas de doble ingreso (Jacobs \& Gerson 2001). Los norteamericanos trabajan semanas más largas con menos tiempo para la vida en el hogar (Fagan 2001), el promedio de edad de las parejas casadas ha aumentado, y las mujeres permanecen sin hijos durante más tiempo, con un mayor porcentaje que simplemente no tiene hijos (Statistics Canada, 2003b; 2005; 2006). 
El tiempo que hombres y mujeres emplean con otros miembros del hogar ha descendido en las últimas dos décadas. Cuando Statistics Canada estudió a hombres y mujeres con trabajo asalariado, encontró que un 23 por ciento de los trabajadores empleaba seis o más horas de un día laborable con otros miembros del hogar en 1985, mientras que sólo un 14 por ciento hacía lo mismo en 2005. Mientras que el tiempo de las mujeres trabajadoras con otros miembros del hogar suponía en promedio 248 minutos de los días laborales en 1986, este indicador descendió un 15 por ciento (39 minutos) hasta un promedio de 209 minutos en 2005. Se produjo un declive ligeramente mayor al 18 por ciento ( 45 minutos) en el caso de los hombres, desde 250 hasta 205 minutos. Un aumento de 8.4 a 8.9 horas ( 30 minutos) en el día típico de trabajo en Canadá es el responsable de la mayor parte de este declive (Turcotte, 2007).

No sólo la gente dedica más tiempo al trabajo asalariado, también dedican menos tiempo al trabajo en el hogar, ayudado por la proliferación de restaurantes baratos, los servicios a domicilio y los electrodomésticos (Michelson, 1985; Robinson \& Godbey, 1997; Cheng et al., 2007). La frecuencia de la gente que dice cenar junto con la familia "se ha reducido en un tercia en los últimos veinte años, desde en torno a un 50 por ciento hasta el 34 por ciento" (Putnam, 2000: 100).

Tomados en su conjunto, estos datos sugieren que la supuesta familia canadiense "tradicional" posterior a la II Guerra Mundial ha cambiado considerablemente a lo largo de los últimos 30 años. Con seguridad, en el modelo tradicional del libro de texto infantil Fun with Dick and Jane (Anonymous, 1940), los maridos y las esposas seguían cada uno su camino: los maridos fuera en el trabajo asalariado para mantener a la familia; mientras las mujeres se quedan en casa para realizar las labores del hogar (también queda representado en la película Pleasantville, Ross, 1998). Raramente se comunicaban mientras estaban separados. Sin embargo, el modelo tradicional también presumía que maridos, esposas e hijos pasarían la mayor parte de su tiempo libre por la tarde-noche y los fines de semana juntos. El resultado es que mientras "el amor conquista el matrimonio" (para citar el subtítulo del libro de Coontz, 2005), maridos, esposas e hijos han estado trazando a diario vidas individualizas en barrios residenciales y ciudades desperdigadas. Los coches de la familia se han multiplicado, dando lugar a un coche para cada adulto (Putnam, 2000), como también ha ocurrido con los aparatos de televisión: mientras que el 28 por ciento de los hogares de los trabajadores canadienses tenía dos o más televisores en 1987, el 63 por ciento tiene múltiples aparatos en 2005 (Turcotte, 2007). Ver la televisión en familia ha dado lugar 
a la televisión personal. De forma más general, el uso y consumo de medios se ha vuelto más personalizado e individual.

Estos cambios interrelacionados en la composición de los hogares norteamericanos, las complejidades del ciclo vital de matrimonios y divorcios, las labores domésticas y el trabajo asalariado, y las decisiones de tener hijos o no (y cuántos en su caso) significan que la naturaleza de dichos hogares es variada, compleja y dinámica. Las complejidades estructurales y demográficas de la vida norteamericana contemporánea han aumentado las tensiones y las oportunidades (Hochschild, 1997). La vida en el hogar se ha acelerado y la gente realiza multitareas o corre de una tarea a la siguiente, sintiendo que tienen demasiado que hacer y demasiado poco tiempo para hacerlo (Menzies, 2005). La contienda entre la vida en casa y la vida en el trabajo es elevada, pues el día de trabajo se ha alargado, los teletrabajadores hacen toda o parte de su tarea en casa, y los directivos y profesionales se llevan trabajo a casa.

Desde principios de los 1990, los sistemas de comunicación personal - los teléfonos móviles y los ordenadores personales en casa y en el trabajo- han facilitado enormemente la capacidad de relación de la gente dentro y fuera de sus casas (Venkatesh et al, 2003; Wellman \& Haythornthwaite, 2002; Lally, 2002). No sólo mucha gente utiliza Internet en el trabajo, sino que una mayoría de los usuarios norteamericanos de Internet conectan desde sus casas (Madden, 2006; CIP, 2005; Statistics Canada, 2005). Por ejemplo, el 72 por ciento de los canadienses hace uso de Internet desde casa, el trabajo u otros lugares. Mientras que la prevalencia de los ordenadores en el hogar ha contribuido a la conectividad, mucha gente no está atado a un solo ordenador personal en casa (o en el trabajo) -o para el caso, a sus líneas telefónicas. Por el contrario, llevan sus comunicaciones con ellos, utilizando teléfonos móviles, ordenadores portátiles, o acceso a Internet desde casa, el trabajo y lugares públicos.

En un momento en el que las TICs han sido domesticadas en el hogar (Haddon, 1992; 2006; Cumming \& Kraut, 2001), estamos especialmente interesados en las implicaciones de las TICS para la interrelación entre el individualismo y la integración funcional dentro de los hogares (Mesch, 2003; Mezaros, 2004). Los sistemas tecnológicos del hogar tienen que ser estudiados en el contexto de las relaciones sociales del hogar. Por otro lado, la ubicuidad de las TICS es tan grande (y nosotros defendemos que tan importante), que es crucial para comprender las relaciones del hogar contemporáneas. 
Utilizamos encuestas y entrevistas para analizar cómo 167 adultos que viven con su pareja en el hogar se mantienen en contacto entre sí ${ }^{3}$. Examinamos si hay diferencias en la forma en la que hombres y mujeres utilizan las TICS en sus casas, negocian cuestiones familiares y comparten información de Internet ${ }^{4}$. Aunque son muchos los factores que han contribuido a la conversión en hogares en red, nos centramos en la comparación de personas con diferentes niveles de uso de Internet, desde nada a un uso muy frecuente. Nuestras preguntas fundamentales son:

- ¿Cómo se comunican entre sí los adultos miembros del hogar?

- ¿Cómo utilizan las TICs los adultos miembros del hogar para organizar y coordinar su tiempo libre y su comportamiento social dentro y fuera de la casa?

- ¿Cómo utilizan las TICs los adultos miembros del hogar para compartir cosas entre sí?

\section{El proyecto “Vidas Conectadas"}

EI lugar: Los datos recogidos para nuestro proyecto Vidas Conectadas corresponden a East York, un área residencial fundamentalmente urbana situada a unos 30-45 minutos del centro de Toronto, con accesos de comunicaciones y transporte adecuados. East York, con una población de 112.054 habitantes en 48.057 hogares (2.3 personas por hogar; según censo canadiense de 2006) ha sido estudiado en dos ocasiones en la era pre-Internet por el NetLab (Wellman, 2001). En muchos aspectos, los residentes de East York reflejan al Canadá anglófono urbano. El 58 por ciento de los encuestados son mujeres, con una media de edad de 45 años. Casi dos tercios (el 62 por ciento) de los encuestados están casados o tienen pareja estable, y el 61 por ciento tienen niños. La mayoría son de clase trabajadora o de clase media: el 43 por ciento de los encuestados tiene un título universitario. El grupo étnico más amplio es británicocanadiense (el 44 por ciento de los encuestados), con algunas minorías visibles que incluyen al 27 por ciento de los encuestados: principalmente los provenientes del Este de Asia y sudasiáticos.

\footnotetext{
${ }^{3}$ Las "parejas" incluyen a matrimonios oficiales y a parejas de hecho, que viven juntos: la Ley de Familia de Ontario (Sección 29) apenas hace distinciones y permite el matrimonio del mismo sexo.

${ }^{4}$ El tamaño de la muestra del estudio y la especificidad geográfica limitan la generalización de nuestros hallazgos de investigación, aunque creemos que representan una situación más amplia. Nuestros análisis se centran principalmente en el género y en el tipo de uso de Internet y no incluye información demográfica del tipo del estatus socioeconómico y el grupo étnico.
} 
Recolección de datos: realizamos un muestreo aleatorio de adultos no débiles de habla inglesa (18+) y obtuvimos 350 encuestas de 32 páginas completas que fueron aplicadas a mano entre julio de 2004 y marzo de 2005, con una tasa de respuesta del 56 por ciento. La encuesta proporciona información sobre cuánta gente en East York utiliza actualmente Internet, sus redes de comunidades personales, implicación comunitaria, actitudes sociales, y relaciones en el hogar. Excepto que se mencione lo contrario, todas las estadísticas utilizadas en este artículo provienen de dicha encuesta.

Los estudiantes doctorales del proyecto Vidas Conectadas llevaron a cabo entrevistas largas en el hogar (2-4 horas), entre febrero y abril de 2005, con una sub-muestra del 25 por ciento de los encuestados. Las entrevistas proporcionaron información sobre el trabajo diario, el tiempo libre, las relaciones en el hogar, las redes sociales, las rutinas sociales y el uso de las TICs (Hogan, Carrasco \& Wellman 2007).

El uso de Internet: Nuestros datos muestran que los residentes de East York utilizan Internet algo más frecuentemente que el canadiense promedio, en consistencia con las mayores tasas de uso de Internet de los canadienses urbanos anglófonos (Fong, et al., 2001; Ekos, 2004). Sólo el 6 por ciento de los encuestados en pareja nunca utilizan Internet desde sus casas (no usuarios, aunque algunos lo utilizan desde el trabajo $u$ otros lugares); el 24 por ciento son usuarios poco frecuentes, conectados a Internet desde casa una o dos horas a la semana (Media $=1.6$ horas por semana); el 35 por ciento son usuarios moderados, conectados de 3 a 7 horas por semana (Media $=4.6$ horas por semana); mientras que el 35 por ciento son usuarios muy activos, conectados más de 7 horas por semana (Media $=20.5$ horas por semana, o casi 3 horas por día).

Los residentes de East York es más probable que se conecten a Internet desde casa entre las seis y las once de la noche; el tiempo en el que habitualmente están en casa después del trabajo. Cuatro quintos (el 82 por ciento) están online durante dicho período. Es menos probable que utilicen Internet entre las cinco y las 8 de la mañana (17 por ciento).

Es llamativo que aproximadamente el mismo porcentaje de no usuarios que usuarios tienen un ordenador en casa, y que la mayoría de los no usuarios tienen más de un ordenador en casa. Incluso aunque los no usuarios no utilizan el ordenador en casa, sus parejas o hijos sí lo hacen. De hecho, más de un tercio (el 37 por ciento) de los encuestados tienen más de un ordenador en casa (Tabla 1 ). 


\begin{tabular}{|c|c|c|c|c|c|c|}
\hline No usuarios en casa & $\begin{array}{c}\text { Poco } \\
1-2 \\
\text { horas }\end{array}$ & $\begin{array}{c}\text { Mod. } \\
\text { 3-7 } \\
\text { horas }\end{array}$ & $\begin{array}{c}\text { Mucho } \\
8+ \\
\text { horas }\end{array}$ & Total & \multicolumn{2}{|c|}{ Significación } \\
\hline Tamaño de la muestra & $\mathrm{n}=10$ & $\mathrm{n}=40$ & $n=58$ & $n=59$ & \multicolumn{2}{|c|}{$\mathrm{N}=167$} \\
\hline Porcentaje del total de usuarios & 6 & 24 & 35 & 35 & & \\
\hline Media de edad & 41 & 44 & 45 & 42 & 43 & .510 \\
\hline $\mathrm{n}=$ & 9 & 39 & 57 & 59 & 164 & \\
\hline$\%$ Mujeres & 75 & 70 & 49 & 53 & 57 & .126 \\
\hline $\mathrm{n}=$ & 8 & 40 & 57 & 59 & 164 & \\
\hline \% con Niños & 90 & 70 & 74 & 78 & 75 & .564 \\
\hline $\mathrm{n}=$ & 10 & 40 & 58 & 59 & 167 & \\
\hline \% Empleados & 80 & 78 & 74 & 55 & 69 & .051 \\
\hline $\mathrm{n}=$ & 10 & 37 & 57 & 58 & 162 & \\
\hline \% Trabajadores en casa & 0 & 23 & 30 & 32 & 27 & .260 \\
\hline $\mathrm{n}=$ & 8 & 31 & 43 & 34 & 116 & \\
\hline $\begin{array}{l}\text { \% con diploma de estudios } \\
\text { Universitarios }\end{array}$ & 56 & 46 & 37 & 32 & 38 & \\
\hline$\%$ con licenciatura universitaria & 11 & 21 & 26 & 25 & 24 & .580 \\
\hline $\mathrm{n}=$ & 9 & 39 & 57 & 59 & 164 & \\
\hline$\%$ con $>1$ ordenador en casa & 67 & 36 & 37 & 33 & 37 & .457 \\
\hline $\mathrm{n}=$ & 6 & 33 & 57 & 57 & 153 & \\
\hline Media de número de años online & 6.2 & 7.2 & 6.6 & 7.9 & 7.2 & .103 \\
\hline $\mathrm{n}=$ & 9 & 37 & 58 & 57 & 161 & \\
\hline
\end{tabular}

Tabla 1. Características de los residentes de East York con pareja por horas de uso de Internet

Más de dos tercios de los encuestados tienen teléfono móvil (68 por ciento). Como ocurre con Internet, es un porcentaje superior al 61 por ciento de adultos canadienses en 2006 (Ipsos, 2006), probablemente porque la cobertura urbana del teléfono móvil es mejor que la cobertura rural.

La mayoría de los residentes de East York están conectados online y hace muchos años que lo están. Raramente son recién llegados al mundo online. La brecha digital se ha estrechado en East York: los cuatro tipos de usuarios (desde los no usuarios a los usuarios muy activos) tienen una media de edad similar, la mayoría tienen al menos un primer ciclo universitario, la mayoría tienen un trabajo asalariado, y cada tipo ha estado online por un promedio de aproximadamente siete años (Tabla 1), excepto los no usuarios (en el hogar) que han estado online ligeramente menos - pese a que lo hayan estado desde el trabajo, bibliotecas, etcétera. Sólo una minoría de usuarios de 
Internet trabaja desde casa, oscilando desde el 23 por ciento de los usuarios poco frecuentes hasta el 32 por ciento de los usuarios muy activos.

Aunque no hay diferencias de género entre cada uno de los tres grupos de usuarios en la cantidad de tiempo empleada online, los no usuarios y los usuarios poco frecuentes es más probable que sean mujeres. Además, las mujeres que no usan Internet casi siempre tienen niños en el hogar y tienen menos educación formal que las mujeres que usan Internet. Dichas diferencias pueden relacionarse con la división doméstica del trabajo, el cuidado de los niños, el cuidado del marido y otras constricciones temporales (Shelton \& John, 1996; Hochschild, 1989; Luxton, 1980).

\section{Comunicar, coordinar y compartir en los hogares en red: ¿Cómo se comunican entre sí los miembros del hogar?}

La voz predomina sobre los dedos: los teléfonos se utilizan más que Internet (Tabla 2). Las líneas telefónicas tradicionales se utilizan más que los teléfonos móviles, y el correo electrónico se utiliza más que la mensajería instantánea (IM). La comunicación a través de Internet no ha reemplazado otros modos de comunicación; las líneas telefónicas tradicionales y los teléfonos móviles son modos integrales a través de los cuáles la gente se conecta entre sí (este hallazgo es consistente con Quan-Haase et al, 2002; Boase et al, 2005; Wellman, Hogan et al, 2006). La principal diferencia de género es que las madres contactan con sus hijos más que los padres.

Líneas telefónicas tradicionales: Siguen siendo el medio más frecuente por el que los residentes de East York se comunican con sus parejas y sus hijos. Los residentes de East York llaman a sus parejas casi cada día laborable: unas 20 veces a la semana. Sin acceso a Internet en casa, los no usuarios de Internet llaman a sus parejas por teléfono casi a diario. Las mujeres que no utilizan Internet en casa son las que hacen más llamadas de teléfono, pero la relación entre el uso de Internet y del teléfono es compleja, sin variaciones significativas por género o por frecuencia de uso de Internet.

La evidencia muestra que Internet se suma al contacto telefónico, en lugar de sustituirlo. Tampoco la cantidad de uso de Internet reduce de forma significativa la cantidad de uso de las líneas telefónicas tradicionales. Los hombres usuarios de Internet hacen uso de la línea telefónica con la misma frecuencia que los hombres no usuarios, mientras que las mujeres usuarias de Internet llaman por teléfono algo menos que las no usuarias. 


\begin{tabular}{|c|c|c|c|c|c|c|}
\hline & & $\begin{array}{c}\text { No } \\
\text { usuarios }\end{array}$ & $\begin{array}{c}\text { Poco } \\
1-2 \\
\text { horas }\end{array}$ & $\begin{array}{l}\text { Moderado } \\
\text { 3-7 horas }\end{array}$ & $\begin{array}{l}\text { Mucho } \\
8+\text { horas }\end{array}$ & Total \\
\hline & \multicolumn{6}{|c|}{ Entre miembros de la pareja } \\
\hline \multirow[t]{2}{*}{ Llamadas telefónicas a la pareja } & Mujeres & 26.0 & 18.5 & 23.2 & 20.4 & 21.0 \\
\hline & Hombres & 18.0 & 18.9 & 18.5 & 23.6 & 20.5 \\
\hline$n=162$ & Total & 22.3 & 18.6 & 20.7 & 21.9 & 20.8 \\
\hline \multirow[t]{2}{*}{ Llamadas de móvil a la pareja } & Mujeres & 11.3 & 10.3 & 13.6 & 17.0 & 13.5 \\
\hline & Hombres & 3.0 & 22.1 & 11.4 & 14.5 & $14.3+$ \\
\hline $\mathrm{n}=132$ & Total & 9.3 & 14.1 & 12.4 & 15.8 & 13.8 \\
\hline \multirow[t]{2}{*}{ Correo electrónico a la pareja } & Mujeres & - & 8.2 & 9.2 & 5.1 & 7.3 \\
\hline & Hombres & - & 9.0 & 4.1 & 9.6 & 7.3 \\
\hline $\mathrm{n}=130$ & Total & - & 8.5 & 6.4 & 7.2 & 7.3 \\
\hline \multirow{2}{*}{$\begin{array}{l}\text { Correos electrónicos a la pareja } \\
\text { cuando están juntos en casa }\end{array}$} & Mujeres & - & 0 & 1.3 & 1.5 & 1 \\
\hline & Hombres & - & 0 & 0 & 1.8 & 1 \\
\hline $\mathrm{n}=122$ & Total & - & $\mathbf{0}$ & $<1$ & 1.6 & 1 \\
\hline \multirow[t]{2}{*}{ I M a la pareja } & Mujeres & - & 4.9 & 4.3 & 1.8 & 3.4 \\
\hline & Hombres & - & 3.3 & 1.9 & 7.0 & 4.3 \\
\hline \multirow[t]{2}{*}{$\mathrm{n}=121$} & Total & - & 4.4 & 2.8 & 4.3 & 3.8 \\
\hline & \multicolumn{6}{|c|}{ Padres - hijos } \\
\hline \multirow[t]{2}{*}{ Llamadas telefónicas a los hijos } & Mujeres & 12.6 & 9.0 & 15.4 & 13.3 & 12.9 \\
\hline & Hombres & 6.0 & 12.0 & 11.7 & 13.0 & 12.3 \\
\hline$n=108$ & Total & 8.7 & 10.0 & 13.4 & 13.2 & 12.6 \\
\hline \multirow[t]{2}{*}{ Llamadas de móvil a los niños } & Mujeres & 7.2 & 7.0 & 8.3 & 9.5 & 8.4 \\
\hline & Hombres & 0 & 9.8 & 3.5 & 7.7 & 6.0 \\
\hline $\mathrm{n}=87$ & Total & 5.1 & 7.7 & 5.8 & 8.6 & 7.3 \\
\hline \multirow[t]{2}{*}{ Correos electrónicos a los hijos } & Mujeres & - & 5.1 & 1.2 & 4.8 & 3.8 \\
\hline & Hombres & - & 6.8 & $<1$ & 2.6 & 2.5 \\
\hline $\mathrm{n}=79$ & Total & - & 5.8 & 1.0 & 3.7 & $3.2+$ \\
\hline \multirow{2}{*}{$\begin{array}{l}\text { Correo electrónico a los hijos } \\
\text { cuando están juntos en el hogar }\end{array}$} & Mujeres & - & $<1$ & 0 & 0 & 0 \\
\hline & Hombres & - & $6.0^{*}$ & 0 & 0 & $<1$ \\
\hline$n=69$ & Total & - & 2.5 & 0 & 0 & $<1+$ \\
\hline \multirow[t]{2}{*}{ I M a los niños } & Mujeres & - & 3.3 & 0 & 0 & 1.1 \\
\hline & Hombres & - & $5.0+$ & 0 & 0 & $1.0+$ \\
\hline$n=73$ & Total & - & 3.9 & 0 & 0 & $1+$ \\
\hline
\end{tabular}

*Indica significación $<\mathrm{p}=.05 ;$ +Indica significación $<\mathrm{p}=.10$; Nunca=0; >Mensualmente=1; En torno a mensualmente=3; En torno a semanalmente=6; En torno a diariamente $=30$.

Tabla 2. Uso promedio de los medios (tiempo mensual) por frecuencia de uso de Internet y género. 
Aunque los usuarios de East York utilizan las líneas telefónicas con menos frecuencia para contactar con sus hijos que con su pareja, las primeras constituyen todavía el medio más frecuente para contactar con los hijos. De modo similar a la comunicación entre miembros de la pareja, la frecuencia del uso de Internet entre padres e hijos no se relaciona de forma significativa con la frecuencia de uso de las llamadas telefónicas. Las madres puede que llamen a sus hijos más que los padres, aunque la diferencia de género no es significativa. Por ejemplo, las mujeres que son usuarias poco frecuentes de Internet llaman a sus hijos un promedio de dos veces a la semana mientras que los hombres llaman más o menos semanalmente.

El teléfono móvil: Los teléfonos móviles son el segundo medio de comunicación entre los miembros del hogar, sólo después de las líneas telefónicas tradicionales (como casi todos los norteamericanos, utilizan los teléfonos móviles para hablar más que para escribir mensajes). Como en el caso de las líneas telefónicas, el uso de Internet no está asociado con un menor uso del teléfono móvil. Tanto los usuarios muy frecuentes como los poco frecuentes llaman a sus parejas con el teléfono móvil casi cada día, mientras que los usuarios moderados llaman desde sus móviles ligeramente menos. Aunque ninguna de estas diferencias es estadísticamente significativa, los no usuarios de Internet usan los móviles al menos para llamar a sus parejas, llamando ligeramente más que una vez a la semana. Hay un patrón similar en las llamadas a los hijos desde el móvil, de forma que tanto los usuarios poco frecuentes de Internet como los muy activos llaman a sus hijos desde el móvil semanalmente, mientras que los no usuarios y los usuarios moderados llaman menos de una vez a la semana. La baja tasa de uso del móvil por parte de los no usuarios de Internet sugiere que más que utilizar el móvil para compensar la no utilización de Internet, algunos evitan tanto el móvil como Internet, posiblemente debido al coste, a la aversión tecnológica o a no percibir la necesidad. Algunos no-usuarios prefieren no utilizar ningún tipo de teléfono (incluyendo las líneas tradicionales) y favorecer en su lugar el contacto cara a cara. Como un participante nos contó en la entrevista:

No soy mucho del teléfono. Prefiero en persona. No soy mucho del teléfono comparado con Irene... que se lleva al teléfono una hora y media... No lo entiendo. Yo cojo el teléfono para quedar. No cuento mi vida por teléfono. Prefiero hablar en persona. (\#852 no usuario de Internet)

Las mujeres suelen utilizar los móviles para llamar a sus parejas y a los hijos más frecuentemente que los hombres. De hecho, los hombres no usuarios de Internet son los que tienen la tasa más bajo de uso del teléfono móvil. El conjunto muestra que los hombres utilizan más los móviles porque los hombres que son usuarios poco 
frecuentes llaman dos veces más frecuentemente que las mujeres. Esta es una diferencia marginalmente significativa, de forma que dichos hombres llaman en promedio casi a diario mientras que las mujeres algo más de una vez por semana.

Correo electrónico: Los miembros del hogar también utilizan el correo electrónico para comunicarse, aunque menos frecuentemente que las líneas telefónicas y los teléfonos móviles: una o dos veces a la semana en promedio. Sin embargo, raramente mandan correos a su pareja cuando ambos están en casa: los usuarios poco frecuentes y los moderados nunca lo hacen mientras que los usuarios muy activos lo hacen menos de una vez al mes 5 .

Se dan menos correos electrónicos a los hijos que comunicación telefónica. Además, raramente nadie manda correos a los hijos cuando están juntos en casa. No hay diferencias de género estadísticamente significativas, pero la Tabla 2 muestra que los hombres mandan correos a sus parejas con la misma frecuencia que las mujeres, mientras que las madres mandan correos a sus hijos con más frecuencia que los padres.

Mensajería instantánea: Varias noticias en los medios han anunciado que los miembros del hogar están utilizando cada vez más la mensajería instantánea (I M) para estar en contacto (por ejemplo, Schwartz, 2004). Presumiblemente, estas historias se dan en respuesta al gran uso de IM entre niños y adolescentes (Lenhart, 2002). Pero lo que es habitual entre los adolescentes y una moda entre los medios, es raro en lo que se refiere a los adultos. En promedio, la gente utiliza IM mucho menos de una vez a la semana, aunque el promedio oculta otras cuestiones: muchos nunca utilizan IM, mientras que unos pocos lo utilizan más frecuentemente. Los padres casi nunca utilizan IM con sus hijos: este es un medio para el Chat entre hijos sólo. Además, raramente alguno de los residentes en East York utiliza IM para comunicarse con sus esposos/parejas o hijos mientras están en casa juntos. Esto podría ocurrir porque los usuarios de IM son por lo general jóvenes adultos y adolescentes, mientras que los residentes de East York son habitualmente de mediana edad.

\footnotetext{
${ }^{5}$ Esto contrasta con la experiencia de los dos autores de este artículo (ambos usuarios muy activos) que envían mensajes a sus familiares a diario, remitiendo a cosas interesantes en la Web, reenviando mensajes de terceros, incluyendo fotos adjuntas, etcétera.
} 


\section{¿Cómo utilizan las TICS los miembros del hogar para organizar su tiempo libre y su comportamiento social?}

Las rutinas, horarios y tiempo libre de los residentes en East York suelen estar completos y ocupados y son complejos y móviles. Además de su implicación rutinaria en el trabajo asalariado o en el trabajo doméstico no pagado, muchos/algunos están también ocupados con hobbies: el 9 por ciento de los participantes son activos en grupos de hobby, el 9 por ciento en asociaciones profesionales, el 13 por ciento en organizaciones religiosas, y el 7 por ciento son activos en ligas deportivas - además de otras actividades de tiempo libre menos organizadas tales como hacer ejercicio, dar paseos y hacer deporte (natación, bicicleta, golf, etcétera). Para aquellos con hijos, hay una responsabilidad añadida que supone dedicar más tiempo a las actividades extracurriculares de sus hijos tales como el deporte, el ballet o los scouts. Para llevar a cabo el día, los miembros del hogar tienen que organizar no sólo su propio programa sino también tomar en consideración el programa de los demás en la casa. Una entrevistada dice que ella se siente "como si fuera un taxi" para los hobbies de sus hijos, conduciendo de una actividad a la siguiente, mientras que un hombre que es usuario moderado escribe correos a su esposas sobre

(...) el horario de los deportes, cosas como: voy aquí, vamos allí; ellos tienen que ir aquí, ellos tienen que ir allí: “¿puedes llevarlos?" ¿Entiendes? Citas con el dentista: "Bien, ahora los estoy llevando al dentista y tal y tal, [así que] haz tal cosa en tu camino al trabajo" (\#455).

Correos electrónicos a los miembros del hogar desde casa: Escribir a la pareja desde casa ayuda a mediar las frenéticas rutinas de los residentes de East York, si bien no todos los hogares son iguales. Los usuarios moderados de Internet escriben correos a su pareja un promedio de una vez por semana, mientras que los usuarios muy activos lo hacen un promedio aproximado de tres veces por semana, mientras que los usuarios de Internet poco frecuentes por lo general no lo remiten correos electrónicos a sus parejas desde casa (Tabla 3). Hay también algunas diferencias de género, aunque los patrones se mezclan y las diferencias no son significativas.

Mantenerse conectados con los miembros del hogar es vital. Pese a que la gente puede utilizar varias herramientas de comunicación, las oportunidades sociales que las TICS y el correo electrónico en particular- proporcionan es importante. La gente móvil y multitarea valoran la facilidad y el carácter práctico del correo electrónico, incluyendo su asincronía y su carácter no intrusivo. A diferencia de las llamadas de teléfono, el corro electrónico no interrumpe a la gente en el trabajo o durante el cuidado de los 
hijos, puede accederse al mismo en cualquier sitio en el que haya conexión a Internet, y puede responderse en los huecos de un día ocupado. Los participantes en las entrevistas señalan de forma consistente cómo el correo electrónico es una forma útil de llevar un registro de lo que los esposos, las parejas y los niños están haciendo. Como dice un usuario moderado:

Es más rápido, cuando tienes un día ocupado, mandar rápidamente un correo diciendo, “¿Te viene bien esto?” en lugar de coger el teléfono y llamar (\#442).

\begin{tabular}{|c|c|c|c|c|c|}
\hline & & $\begin{array}{c}\text { Poco } \\
1-2 \\
\text { horas }\end{array}$ & $\begin{array}{l}\text { Mod. 3-7 } \\
\text { horas }\end{array}$ & $\begin{array}{l}\text { Mucho } \\
8+\text { horas }\end{array}$ & Sig. \\
\hline \multirow{6}{*}{$\begin{array}{l}\text { Número medio de correos-e enviados a } \\
\text { miembros del hogar desde casa }\end{array}$} & Mujeres & 0 & 2.3 & 2.4 & .093 \\
\hline & $n=$ & 26 & 28 & 31 & \\
\hline & Hombres & 1.1 & 0 & 3.1 & .004 \\
\hline & $\mathrm{n}=$ & 11 & 27 & 28 & \\
\hline & Total & $<1$ & 1.4 & 2.8 & .010 \\
\hline & $n=$ & 37 & 56 & 59 & \\
\hline \multicolumn{6}{|l|}{$\operatorname{sig}=.905$} \\
\hline \multirow{6}{*}{$\begin{array}{l}\text { Número medio de correos-e enviados a } \\
\text { miembros del hogar desde el trabajo }\end{array}$} & Mujeres & 4.0 & 5.1 & 0 & .342 \\
\hline & $n=$ & 17 & 17 & 10 & \\
\hline & Hombres & 1.7 & 1.1 & 2.7 & .369 \\
\hline & $n=$ & 7 & 16 & 13 & \\
\hline & Total & 3.3 & 3.2 & 1.8 & .634 \\
\hline & $n=$ & 24 & 33 & 23 & \\
\hline $\mathrm{sig}=.202$ & & & & & \\
\hline
\end{tabular}

Tabla 3. Número medio de correos electrónicos enviados de casa al trabajo por semana (por frecuencia de uso de Internet y género)

No hay duda de que los miembros del hogar se mantienen conectados cuando están separados. Pese a que los residentes de East York pueden ocasionalmente viajar para encontrarse cara a cara, y con frecuencia utilizan líneas telefónicas, móviles, IM o correo electrónico para contactar, algunos utilizan deliberadamente el correo electrónico como el medio de su elección. Un usuario moderado describe su elección de los medios:

I: ¿Alguna vez mandas correos electrónicos a tu marido desde aquí? ¿Y le mandas mensajes a su trabajo? 
P: Es lo habitual porque cuando estamos aquí, hay cientos de cosas que hacer y se termina el día; las niñas están en la escuela, él camino de su trabajo, Adam está durmiendo, y pienso, "OK, necesitamos hacer esto, esto, esto y esto". Así que no voy a coger el teléfono, porque está en el trabajo y no quiero hacerlo, pero voy a enviarlo y de ese modo ya está fuera de mi cabeza. Ya lo he comunicado y cuando nos vemos por la noche, "ah, sí, ese correo que me enviaste" $(\# 421)^{6}$.

Mandar correos a los miembros del hogar desde el trabajo: Pese a que los usuarios poco frecuentes de Internet raramente mandan correos a sus parejas desde casa, mandan un promedio de tres mensajes a la semana desde el trabajo. Aunque los usuarios muy activos de Internet mandan correos desde el trabajo ligeramente con menos frecuencia (un promedio de dos por semana) que desde casa, cuando se combinan ambos, los usuarios muy activos son lo que mandan correos con más frecuencia: un promedio de 4.6 mensajes por semana. Las mujeres mandas correos a los miembros del hogar desde el trabajo más que los hombres, incluso pese a que es frecuente que trabajen menos horas por semana (33) que los hombres (44). Pese a que el día de trabajo ha aumentado a lo largo de los últimos veinte años, también lo ha hecho la extensión de las pausas para comer o para el café (Statistics Canada, 2005).

El correo electrónico ayuda a los miembros del hogar a permanecer en contacto en el momento álgido del día de trabajo, especialmente cuando el trabajo podría interferir con la comunicación entre la pareja. Uno de los hombres usuarios muy activos señala que él comunica a su mujer a través del correo que está en una reunión y no está disponible:

Es práctico: da menos problemas, distrae menos. Ella no sabe cuando estoy en una reunión o cosas por el estilo... (\#561).

De un modo parecido, un usuario poco frecuente señala:

Yo lo hago desde el trabajo todo el tiempo - a mi marido en particular, porque mis hijos durante el día no están online, sino en la escuela. Pero a mi marido... Sé que él pasa una gran parte del día delante del ordenador. No todo el día, pero sí mucho, y sé que lee el correo con frecuencia, de forma que puede pillarlo normalmente ahí. No es que no tenga un móvil con él todo el tiempo y cosas como esa, sino que prefiero mandarle un correo... Le escribí un correo hoy, ni siquiera sé de qué iba - algo así como "¿vas a venir a casa después de la escuela para sacar el perro?" Ya sabes, ese tipo de cosas (\#432).

\footnotetext{
6 "E" se refiere al Entrevistador y "P" se refiere al Participante. El número detrás de cada cita es el número de identidad de dicho participante en el estudio de Vidas Conectadas.
} 


\section{¿Cómo utilizan los miembros del hogar las TI Cs para compartir cosas entre sí?}

Compartir experiencias online con la pareja: En los viejos tiempos las familias solían reunirse en torno al piano. Más recientemente las familias se reunían para ver un programa de televisión. Ahora muchas familias de East York se reúnen para usar Internet juntos en casa. Los usuarios muy activos de Internet emplean un promedio aproximado de tres horas por semana con sus parejas usando Internet (Tabla 4). Los usuarios moderados pasan un promedio de en torno a una hora y media online con sus parejas, mientras que los usuarios poco frecuentes hace uso un promedio de una hora por semana online con su pareja.

Mientras más se usa Internet, más tiempo pasa la pareja junta online: los usuarios muy activos pasan 1.8 veces más online con sus parejas que los usuarios moderados y 2.3 veces más que los usuarios poco frecuentes. No obstante, los usuarios menos frecuentes pasan proporcionalmente más parte de su tiempo online con sus parejas que los usuarios muy activos: Tres cuartos ( 75 por ciento) del tiempo online de los usuarios poco frecuentes es con sus parejas, en comparación con un tercio ( 35 por ciento) de los usuarios moderados y un sexto (14 por ciento) de los usuarios muy activos. Cuando la gente pasa más tiempo online, las interacciones del hogar aumentan pero no a una tasa tan alta como las interacciones que no incluyen compartir con la pareja en casa.

Los residentes de East York utilizan Internet para mostrar o compartir cosas de interés. A veces usan Internet juntos cuando planifican actividades, tales como películas, conciertos y vacaciones - o incluso sólo por diversión, aunque también lo utilizan para complementar otras actividades de tiempo libre que hacen juntos, como ver televisión. (Los usuarios muy activos, moderados y poco frecuentes también pasan en promedio tres horas por semana viendo televisión con sus parejas).

Una de las usuarias muy activas explica cómo el tiempo que ella y su marido pasan online con cosas de interés hace que se reúnan para hacer uso conjunto de Internet:

Mi marido: la única cosa que él hace en Internet es mirar la oferta inmobiliaria, en todos sitios. El otro día por la noche estuvimos los dos juntos sentados mirando apartamentos en México. ¡Hagámoslo! Sabes, hemos mirado propiedades en Grecia, pero era ridículo... con frecuencia hacemos eso... A él también le encanta mirar muscle cars; ¿sabes?, los coches pasados de moda. Él y mi hijo mayor se sientan a mirar ese tipo de cosas en Internet. Así que, efectivamente, a veces nos sentamos juntos, principalmente para eso, para mirar la oferta inmobiliaria, en plan “¡Mira esas casas!” y tal y mirar. (\#174) 


\begin{tabular}{|c|c|c|c|c|c|}
\hline & & $\begin{array}{c}\text { Poco } \\
1-2 \\
\text { horas }\end{array}$ & $\begin{array}{c}\text { Mod. } \\
3-7 \\
\text { horas }\end{array}$ & $\begin{array}{l}\text { Mucho } \\
8+\text { horas }\end{array}$ & Sig. \\
\hline \multirow{6}{*}{$\begin{array}{l}\text { Número medio de horas por semana que pasa } \\
\text { con su pareja online }\end{array}$} & Mujeres & 1.2 & 1.5 & 3.1 & .103 \\
\hline & $\mathrm{n}=$ & 26 & 26 & 31 & \\
\hline & Hombres & 1.2 & 1.7 & 2.6 & .306 \\
\hline & $n=$ & 12 & 29 & 28 & \\
\hline & Total & 1.2 & 1.6 & 2.8 & .031 \\
\hline & $n=$ & 38 & 56 & 59 & \\
\hline \multicolumn{6}{|c|}{$\operatorname{sig}=.784$} \\
\hline \multirow{6}{*}{$\begin{array}{l}\text { Número medio de horas por semana que pasa } \\
\text { con sus hijos online }\end{array}$} & Mujeres & $2.1^{7}$ & 2.2 & 4.6 & 201 \\
\hline & $n=$ & 17 & 19 & 24 & \\
\hline & Hombres & 1.5 & 1.3 & 1.5 & .933 \\
\hline & $n=$ & 6 & 24 & 20 & \\
\hline & Total & 2.0 & 1.7 & 3.2 & .203 \\
\hline & $\mathrm{n}=$ & 23 & 43 & 44 & \\
\hline
\end{tabular}

Tabla 4. Número medio de horas que pasan con la pareja y los hijos online por semana (por frecuencia de uso de Internet y género)

De modo similar, un usuario moderado cuenta que él y su esposa buscan información sobre viajes online:

Especialmente, cuando suena a anuncio de televisión sobre viajes; comentamos “¿qué te parece este hotel?" (\#844)

Los miembros del hogar utilizan Internet juntos no sólo para explorar y compartir intereses personales, sino también para ayudar en la realización de tareas, como en la renovación de la casa. Un usuario muy activo explica:

Estamos investigando juntos varias cosas que nos gustan a los dos; estamos haciendo una reforma e investigamos qué tipo de lavabo comprar... (\#383)

Un usuario moderado habla de un programa de televisión que les gusta ver juntos y cómo revierte en el uso conjunto de Internet:

Investigamos cualquier cosa que necesitamos para tomar una decisión, como comparar coches o... nosotros somos muy fans de "Amazing Race"... y después del programa nos vamos a Internet y buscamos clips sobre lo próximo para intentar elegir. (\#421)

\footnotetext{
${ }^{7}$ Se pidió a los participantes que eligieran una categoría de las horas que emplean en lo siguiente. Las categorías (tales como 1-4) fueron codificadas en su término medio (2.5) y puede explicar por qué parece que los usuarios poco frecuentes pasan todo el tiempo online con sus hijos. La propia pregunta puede ser problemática porque los participantes podrían incluir el tiempo pasado con sus hijos en otros lugares (tales como una librería u otros miembros familiares).
} 
Además de compartir información entre sí, los miembros del hogar utilizan con frecuencia Internet para comunicarse juntos con sus familiares, tanto local como globalmente, utilizando IM, el teléfono a través de Internet y Webcams. Un usuario moderado comenta que él chatea online con los familiares que viven lejos, mientras que su esposa le acompaña:

Cuando chateo con mi familia, mi mujer se sienta conmigo. Ella también chatea con ellos. Yo chateo con su familia también. Entonces, ella se sienta conmigo y chatea con su familia (\#343).

Compartiendo experiencias online con los hijos: Abundan las historias sobre cómo los niños se han vuelto expertos en ordenadores, a veces aislándose de forma que puedan comunicarse con sus amigos a través de IM O navegar a través de MySpace (Shiu \& Lenhart, 2004; boyd, 2006). Aunque hay mucho de verdad en estas historias, sólo cuentan parte de la historia. Las parejas pasan tiempo online con sus hijos, igual que lo hacen entre ellos. Las mujeres pasan más tiempo online con sus hijos que los hombres (Tabla 4). Son especialmente destacables las mujeres que son usuarias muy activas de Internet, que pasan en promedio 4.6 horas semanales online con sus hijos, comparadas con los hombres usuarios muy activos que pasan como mucho un tercio de ese tiempo, 1.5 horas por semana.

El uso de Internet con los hijos es una nueva forma de pasar tiempo con los niños, y frecuentemente se relaciona con las responsabilidades domésticas y de cuidados personales de las mujeres. Como señala una usuaria poco frecuente:

Bueno, vamos a Treehouse TV [un sitio Web]. Es algo así como la cadena de televisión de los niños: tienen un sitio Web con juegos y música... Si estoy online y ella llega, escribo Treehouse TV y busco alguna cosa con ella. (\#263)

Las madres no sólo pasan tiempo online con sus hijos en su tiempo libre. También utilizan Internet instrumentalmente, como una herramienta educativa y de aprendizaje con sus hijos. Una usuaria muy activa explica:

Tenemos acceso al sitio de Winnie the Pooh para contar, para el alfabeto y ese tipo de cosas. Este tipo de juegos educativos no son juegos juegos, sino que es para aprender a contar o el alfabeto, o ya sabes: Dora Exploradora - con música infantil y demás. (\#341)

Los padres pasan tiempo con los hijos mayores - incluso con los adultos- igual que con los más pequeños. Una usuaria muy activa comenta:

Mi hijo más pequeño [adulto] y yo pasamos más tiempo buscando cosas fascinantes en el ordenador del tipo " $i$ Oh, vamos... mira esto! ¿Sabes? Cualquier cosa. Algunas veces nos sentamos uno al lado del otro delante del ordenador y hacemos cosas (\#174). 
Utilizar Internet juntos o al mismo tiempo permite a los miembros del hogar pasar tiempo juntos, incluso aunque estén haciendo cosas diferentes ${ }^{8}$. Como ha sido el caso con la Televisión (Silverstone, 1994), hacer un uso común de Internet es mucho más fácil cuando los ordenadores están localizados en lugares de la casa que conducen a la interacción social. Casi la mitad (el 43 por ciento) de los residentes de East York que viven en pareja dicen tener acceso a Internet en áreas comunes, aunque es mayor el número de casos (el 64 por ciento) en los que los puntos de acceso a Internet están en espacios más segregados, tales como oficinas y habitaciones personales ${ }^{9}$. Las áreas comunes - tales como el comedor, el salón familiar, la salita de estar, la habitación de invitados, el sótano o las áreas recreativas- no sólo proporcionan un acceso fácil a Internet, también facilitan una comunicación fácil con otros miembros del hogar, conformando un espacio que congrega a la gente ${ }^{10}$ :

Hay [sólo] una mesa de despacho, pero hay otra silla que podemos acercar. Habitualmente hacemos eso, y es también donde tenemos los juguetes de los niños y otras cosas en el otro lado de la habitación. Siempre estamos saltando por encima de los juguetes para hacer eso (\#421 usuario moderado).

Compartir información: Mientras que algunos residentes de East York pasan tiempo online junto con sus parejas o sus hijos, otros no lo hacen. Estar online en casa puede ser una actividad solitaria; conectar con amigos y familiares, buscar información, hacer gestiones bancarias y demás son cosas que la gente normalmente hace por sí mismos. Sin embargo, tener Internet en el hogar también ayuda a compartir la información que la gente encuentra online. Muchos entrevistados se refieren al escenario del tipo "eh, ¡mira esto!", cuando encuentran algo interesante online y quieren mostrarlo y compartirlo con otra persona, ya sea en persona, a través de IM o por correo electrónico. Por ejemplo:

Si aparece algo interesante, como que en las noticias aparezca algo relacionado con un lugar que hemos visitado en uno de nuestros viajes, la llamo y le digo: "mira esta historia", o algo de eso. (\#815 usuario muy activo)

[Mi marido] a veces me lo imprime o me lo enseña. Otras veces simplemente habla de lo que ha encontrado. (\#832 usuario moderado)

\footnotetext{
${ }^{8}$ Véase la discusión de Bianchi, Robinson \& Milkie's (2006) de cómo los padres realizan multi-tareas e incorporan a los niños en sus actividades de tiempo libre. También afirman que los padres están dedicando tanto tiempo a sus hijos (si no más) que los padres en 1965.

${ }^{9}$ Los porcentajes están por encima del 100 por ciento porque muchas casas tienen dos ordenadores, y mucha gente utiliza ordenadores en casa y en el trabajo.

${ }^{10}$ Hemos observado esto en nuestra experiencia también: uno de los autores escribió este artículo sentado al lado de su esposa, mientras el otro lo escribió al lado de su hijo, ambos con dos ordenadores en casa.
} 
En las casas con más de un ordenador, la gente se manda notas interesantes y enlaces Web entre sí. Uno de los pocos participantes que es usuario de I Ming lo utiliza para mandar alertas a otros miembros del hogar, "si hay algo que quiero que mi mujer o mis hijos vean" (\#883). Un usuario moderado comenta:

Hacemos un montón de cosas en paralelo y entonces traemos los resultados y decimos: "mira, iAdivina lo que he encontrado!" o "no encuentro nada, ¿puedes echarme una mano?". Sabes... "Dijiste que encontraste algo interesante la última vez, ¿Dónde lo encontraste? Ese tipo de cosas (\#373).

\section{Redes de hogares}

\section{Expertos en múltiples medios}

Mientras que algunos comentaristas (por ejemplo, Nie, Hillygus \& Erbring, 2002) han sugerido que el uso de los ordenadores es en parte responsable del declive en la interacción social entre los miembros de los hogares, la evidencia sistemática pone en duda esta afirmación (Katz \& Rice 2002; Gershuny 2003; Mesch 2006). Como estos otros estudios, hemos encontrado que Internet no reemplaza al contacto cara a cara con familiares o amigos. Por el contrario, se suma al mismo y lo enriquece.

Las complejas vidas de los residentes en East York - combinadas con la movilidad personal y la conectividad móvil- significan que la mayoría de la gente usa Internet y los teléfonos para orquestar las tareas y programas de su hogar. La amplia disponibilidad de la conexión a Internet -junto con la evolución de los dispositivos de comunicación e información- los habilita para utilizarla de muy diferentes formas: desde la comunicación con familiares y amigos y la obtención de información general, a tareas más específicas al contexto tales como la búsqueda de información sanitaria para sus hijos, la búsqueda de recetas para cenar y la planificación de las vacaciones familiares (Kayahara \& Wellman, 2007; Kennedy, 2007). La comunicación - por correo electrónico e IM- y la búsqueda de información en la Web son asuntos tanto individuales como del hogar. Los residentes en East York se comunican frecuentemente con sus parejas e hijos.

Pese al amplio interés generado por Internet y los teléfonos móviles, las líneas telefónicas siguen siendo el modo de comunicación preeminente. Los teléfonos móviles son los siguientes más utilizados por los residentes de East York, seguidos por el correo electrónico y el IM. Hay pocas diferencias de género en cómo -y con qué frecuencia- se utiliza Internet, excepto en el hecho de que las madres han trasladado sus roles tradicionales de cuidado de los hijos a Internet. 
Se podría suponer que la ausencia de Internet en el hogar animaría a los usuarios a hacer uso del teléfono en mayor medida para estar conectados. Pero, para nuestra sorpresa, los no usuarios de Internet no utilizan las líneas telefónicas o el teléfono móvil con más frecuencia que los usuarios de Internet, y algunos tienen aversión a la utilización de otra forma de TIC: Ios teléfonos móviles.

Excepto para dichos no usuarios, la multiplicidad de medios es la norma. Los residentes de East York usan cualquier medio que tengan a mano y que sea apropiado para contactar con sus parejas o sus hijos en casa, en el trabajo, o en movimiento (Haythornthwaite \& Wellman, 1998). Los miembros del hogar tienen vidas complejas y ricas en medios. Todos excepto unos cuantos no usuarios utilizan el correo electrónico para comunicarse con sus parejas y con los hijos, valorando el carácter asíncrono y no intrusivo.

Los usuarios poco frecuentes muestran algunos resultados no esperados. Mientras que pasan menos tiempo en Internet en su conjunto, utilizan Internet para comunicarse con los miembros del hogar en mayor medida que los usuarios muy activos. Mandan correos electrónicos a sus parejas en mayor medida que otros grupos, utilizan IM con sus parejas con más frecuencia, envían correos electrónicos a sus hijos - tanto cuando están bajo el mismo techo como en otras circunstancias- y utilizan IM con sus hijos también con más frecuencia. Los hogares siguen siendo el núcleo de los usuarios poco frecuentes de Internet, mientras que entre los usuarios que van de moderados a muy activos utilizan Internet para obtener información y comunicación con el exterior, a partir de su base de comunicación en el hogar.

Este patrón de uso poco frecuente de Internet centrado en el contacto con familiares y amigos se relaciona en parte con patrones de comunicación diferenciados por género. El 70 por ciento de los usuarios poco frecuentes de Internet son mujeres. La investigación previa ha mostrado que las mujeres tienden a utilizar Internet para reforzar las relaciones personales existentes con familiares y amigos (Boneva \& Kraut, 2002; Kennedy, Wellman \& Klement, 2003; Shade, 2004). De un modo similar al uso que las mujeres han hecho del teléfono (Rakow, 1992; Moyal, 1992: Fischer, 1992), la comunicación a través de Internet permite a los residentes de East York que están en casa, preeminentemente mujeres, superar el aislamiento (véase también Miyata, 2002). 
Aunque los usuarios de ordenadores son a veces representados con los ojos clavados en sus pantallas sin mirar a su alredor, nuestra investigación ha encontrado una gran cantidad de uso compartido de Internet en los hogares de East York. Los miembros del hogar no sólo se envían mensajes y enlaces a Webs de interés, sino que sientan juntos para compartir información y para comunicarse juntos con la familia y los amigos. Con frecuencia es más fácil y más práctico hacer esto con el correo electrónico que vía telefónica o en persona. La búsqueda de información online con frecuencia estimula la comunicación entre los miembros del hogar. Bien se sientan juntos, o le piden a su esposo o hijos que miren algo por ellos, o bien pasan espontáneamente la información que han encontrado online. La información que comparten se refiere frecuentemente a cuestiones inmobiliarias, viajes y vacaciones, reparaciones o renovaciones en el hogar, o información sobre productos. Es un intercambio de información, una socialización del capital de conocimiento y un facilitador de la integración funcional entre los miembros del hogar.

\section{Hacia el individualismo en red}

La combinación de coches conducidos personalmente con TICs personalizadas - tales como Internet y los teléfonos móviles- ayuda a los residentes de East York a negociar las complejidades de sus rutinas, horarios y vida familiar. Están frecuentemente en movimiento: mediando y negociando las actividades laborales, escolares, maritales, parentales, organizativas y demás relaciones sociales. El resultado es simultáneamente una menor conectividad en persona en el hogar y una mayor conectividad a través de las TICs. Pese a que las TICs permiten la separación física de los miembros del hogar, también mantienen a la gente conectada y en red cuando se comunican social e instrumentalmente, mientras coordinan los horarios y las tareas del hogar.

Los hogares de East York son ahora los ejes de redes de comunicación individualizadas en lugar de casas autónomas a las que sólo se accede a través del timbre, las líneas telefónicas y el correo ordinario. Con un uso frecuente y una amplia difusión, las TICS se han convertido en un medio clave a través del cual la mayoría de los hogares se comunican y coordinan un frenético programa de actividades. Puesto que el uso de Internet complementa el contacto telefónico y en persona, la gente está más conectada que nunca, y utilizan habitualmente Internet para equilibrar y organizar las tareas de la vida diaria. 
Aunque los residentes de East York raramente informan de mantener comunicaciones en secreto respecto a los miembros del hogar, cada persona tiene más autonomía en la comunicación, el establecimiento de la agenda, e incluso en la toma de decisiones sobre que TICS adoptar y utilizar. Su teléfono móvil es personal, a diferencia de la línea telefónica del hogar. Incluso cuando hacen uso de un ordenador, acceden personalmente al correo electrónico y al IM. Pueden comunicarse con otros y negociar sus propios planes y horarios con menor probabilidad de que otros miembros del hogar sepan cuándo y con quién se están comunicando. Mandan correos, chatean, telefonean o remiten un mensaje a la dirección de Internet o a un número de móvil sin saber dónde está la otra persona.

Podría parecer que debido a la ubicuidad de las TICS en los hogares actuales, hay menos actividades en común entre los miembros del hogar. Sin embargo, nuestros datos muestran que pese al frecuente uso individual de Internet en casa, los residentes de East York muestran y comparten lo que encuentran online con otro y que también pasan tiempo juntos online. Hay una gran cantidad de comunicación entre los miembros del hogar, tanta que se llevan la comunicación y los problemas del hogar con ellos a lo largo del día. Las TICS no reemplazan al contacto en persona (o a través de línea telefónica) entre los miembros del hogar. Más bien complementan dicho contacto, llenando los huecos a lo largo del día y ayudando a hacer ajustes. Con frecuencia reúnen a los miembros del hogar pasando tiempo juntos online y compartiendo información.

¿Estaban los hogares de los residentes de East York en red antes de las TICs? Algunos lo estaban sin lugar a dudas (Wellman \& Wortley, 1990). Pero las TICS han permitido paradójicamente que los miembros del hogar tengan la capacidad de seguir sus caminos separados mientras permanecen más conectados. De ese modo, en lugar de desmembrar los hogares, las TICs han permitido la percepción, la integración y el apoyo mutuos.

\section{Referencias}

Anonymous. (1940). Fun with Dick and Jane. Scott, Foresman, New York.

Bianchi, S., Robinson, J. \& Milkie, M. (2006). The Changing Rhythms of American Family Life, Russell Sage Foundation, New York.

Boase, J., Horrigan, J., Wellman, B., \& Rainie, L. (2005). The Strength of Internet Ties, Pew Internet and American Life Project, Washington,. www. pewinternet.org 
Bott, E. (1957). Family and Social Network. Tavistock., London.

Boneva, B. \& Kraut, R. (2002). 'Email, gender and personal relationships', The Internet in Everyday Life, eds. B. Wellman and C Haythornthwaite, Blackwell, Oxford, pp. 372403.

boyd, d. (2006). 'Friends, friendsters, and MySpace Top 8', First Monday, vol. 11, no.12, http://www.firstmonday.org/issues/issue11_12/boyd/index.html

Cheng, S.-L., Olsen, W., Southerton, D. \& Warde, A. (2007). 'The changing practice of eating: evidence from UK time diaries, 1975-2000', British Journal of Sociology, vol. 58, no. 1 , pp. 39-50.

Clinton, H. (1996). It Takes a Village. Simon \& Schuster, New York.

Coontz, S. (2005). Marriage, a History. Penguin, New York.

Cumming, J., \& Kraut, R. (2001). Domesticating computer and the Internet, The Information society, vol. 18, no. 3, pp. 221-231.

Ekos (2004). The Dual Digital Divide IV. Ekos Research Associates, Ottawa.

Fagan, C. (2001). 'The temporal reorganization of employment and the household rhythm of work schedules', American Behavioral Scientist, vol. 44, no. 7, pp. 1199-1212.

Fischer, C. (1992). America Calling: A Social History of the Telephone to 1940. University of California Press, Berkeley.

Fong, E., Wellman, B., Wilkes, R. \& Kew, M. (2001). 'Is there a double digital divide? Neighbourhood and individual effects in access to the internet', Proceedings of Urban and Regional Development Conference, International Sociological Association. Amsterdam.

Gershuny J. (2003). Web-use and net-nerds: a neo-functionalist analysis of the impact of information technology in the home. Institute for Social and Economic Research, University of Essex, Colchester, UK.

Haddon, L. (1992). 'Explaining ICT consumption: The case of the home computer', Consuming Technologies, eds. R. Silverstone and E. Hirsch, Routledge, London, pp. 82-96.

Haddon, L. (2006). 'The contribution of domestication research to in-home computing and media consumption', The Information Society, vol. 22, no. 4, pp. 195-203. 
Haythornthwaite, C., \& Wellman, B. (1998). 'Work, friendship and media use for information exchange in a networked organization', Journal of the American Society for Information Science, vol. 49, no. 12, pp. 1101-14.

Hochschild, A.R. (1989). The Second Shift, Viking Penguin, New York.

Hochschild, A. (1997). The Time Bind, Metropolitan, New York.

Hogan, B., Carrasco, J.A. \& Wellman, B. (2007). 'Visualizing personal networks', Field Methods, vol. 19, no. 2, May: in press.

Ipsos Insight. (2006). 'Mobile phones could soon rival the PC as world's dominant internet platform'. April 18.

URL: http://www.ipsos-na.com/news/pressrelease. $\mathrm{cfm}$ ?id=3049

Jacobs, J.A., \& Gerson, K. (2001). 'Overworked individuals or overworked families? explaining trends in work, leisure, and family time', Work and Occupations, vol. 28, no. 1, pp. 40-63.

Katz J. \& Rice, R. (2002). Social Consequences of Internet Use. MIT Press, Cambridge, MA.

Kayahara, J. \& Wellman, B. (2007). 'Searching for culture high and low', Journal of Computer Mediated Communication, vol. 12, no. 4, www.jcmc.edu/vol12/issue3/

Kennedy, T., Wellman, B. \& Klement, K. (2003). 'Gendering the digital divide,' IT \& Society, vol. 1, no. 5, pp. 149-172.

Kennedy, T. (2007). 'Working @ home: negotiating space \& place', in ICTs and Emerging Business Practices, eds. Y. Washida \& S. van der Graaf, Idea, Hershey, PA, pp. 257-279. Lally, E. (2002). At Home with Computers. Berg, Oxford.

Lenhart, A. (2002). 'Teenage life online', Pew Internet \& American Life Project. URL: www. pewinternet.org/pdfs/PIP_Teens_Report.pdf

Luxton, M. (1980). More Than a Labour of Love. Women's Press, Toronto.

Madden, M. (2006). 'Internet penetration and impact', Pew Internet \& American Life Project. URL: http://www. pewinternet.org/PPF/r/182/report_display.asp.

Mesch, G. (2003). 'The family and the Internet', Social Science Quarterly, vol. 84, no. 4, pp. 1038-1050. 
Mesch, G. (2006). 'Family relations and the Internet', Journal of Family Communication, vol. 6, no. 2, pp. 119-138.

Menzies, H. (2005). No Time, Douglas \& Mclntyre., Vancouver.

Mezaros, P. (2004). 'The wired family living digitally in the postinformation age', American Behavioral Scientist, vol. 48, no. 4, pp. 377-390.

Michelson, W. (1985). From Sun to Sun. Oxford University Press, New York.

Miyata, K. (2002). 'Social supports for Japanese mothers online and offline', The Internet in Everyday Life, eds. B. Wellman \& C. Haythornthwaite, Blackwell, Oxford, pp 520-548.

Moyal, A. (1992). 'The gendered use of the telephone', Media, Culture \& Society, vol.14, no. 1 , pp. 51-72.

Nie, N., Hillygus, D. S., \& Erbring, L. (2002). 'Internet use, interpersonal relations and sociability', The Internet in Everyday Life, eds. B. Wellman \& C. Haythornthwaite, Blackwell, Oxford, pp. 215-243.

Quan-Haase, A., Wellman, B., \& Hampton, K. N. (2002). "Capitalizing on the net', The Internet in Everyday Life, eds. B. Wellman \& C. Haythornthwaite, Blackwell, Oxford, pp. 291-324.

Rakow, L. (1992). Gender on the Line, University of Illinois Press, Urbana.

Robinson, J.P, \& Godbey, G. (1997). Time for Life, Pennsylvania State University Press, University Park.

Ross, G. (1998). Pleasantville. Hollywood: Larger Than Life.

Schwartz, J. (2004). 'That parent-child conversation is becoming instant and online', New York Times, January 3.

Shade, L. (2004). 'Bending gender into the net,' Society Online, eds. P.N. Howard \& S. Jones, Sage, Thousand Oaks, CA, pp. 57-83.

Shelton, B.A., \& John, D. (1996). 'The division of household labor', Annual Review of Sociology, vol. 22, pp. 299-322.

Shiu, E., \& Lenhart, A. (2004). 'How Americans use instant messaging'. Washington: Pew Internet \& American Life Project.

http://www.pewinternet.org/pdfs/PIP_Instantmessage_Report.pdf. 
Silverstone, R. (1994). Television and Everyday Life. Routledge, London.

Statistics Canada (2001). 'Census: Families \& Households Profile', Ottawa.

http://www12.statcan.ca/english/census01/Products/Analytic/companion/fam/canada. $\underline{\mathrm{cfm}}$

Statistics Canada (2003a). 'Divorce', Ottawa.

http://www.statcan.ca/Daily/English/050309/d050309b.htm

Statistics Canada (2003b). 'Births', Ottawa.

http://www.statcan.ca/english/freepub/84F0210XIE/2003000/bfront1.htm

Statistics Canada (2005). 'Labour force and participation rates by sex and age group', Ottawa.

http://www40.statcan.ca/l01/cst01/labor05.htm

Statistics Canada (2006). 'Women in Canada', Ottawa.

http://www.statcan.ca/english/freepub/89-503-XIE/0010589-503-XIE.pdf

Turcotte, M. (2007). 'Time spent with family during a typical workday, 1986-2005', Canadian Social Trends, No. 83 (February 13), pp. 2-11.

Venkatesh. A., Kruse, E., \& Shih, E. C. F. (2003). 'The Networked Home', Cognition, Technology and Work, vol. 5, no. 1, p. 23-32.

Wellman, B. (2001).'Physical place and cyberspace', International Urban and Regional Research, vol. 25, no. 2, pp. 227-252.

Wellman, B., \& Haythornthwaite, C. (eds.) (2002). The Internet in Everyday Life, Blackwell, Oxford.

Wellman, B. \& Hogan, B. with Berg, K., Boase, J., Carrasco, J., Côté, R., Kayahara, J., Kennedy, T. \& Tran, P. (2006). 'Connected Lives: The Project', in Networked Neighbourhoods, ed. P. Purcell, Springer, Guildford, UK: pp. 157-211.

Wellman, B. \& Wortley, S. (1990). 'Different strokes from different folks.' American Journal of Sociology, vol. 96, no. 3, pp. 558-88.

Zamaria, C., Caron, A. \& Fletcher, F. (2005). 'Canada online'. Canadian Internet Project Report, Ryerson University, Toronto, pp 236. 